\title{
GENERALIZED VECTOR VALUED DOUBLE SEQUENCE SPACE USING MODULUS FUNCTION
}

\author{
ANINDITA BASU AND P. D. SRIVASTAVA
}

\begin{abstract}
In this paper, we introduce a generalized vector valued paranormed double sequence space $F^{2}(E, p, f, s)$, using modulus function $f$, where $p=\left(p_{n k}\right)$ is a sequence of non-negative real numbers, $s \geq 0$ and the elements are chosen from a seminormed space $\left(E, q_{E}\right)$. Results regarding completeness, normality, $K_{2}$-space, co-ordinatewise convergence etc. are derived. Further, a study of multiplier sets, ideals, notion of statistical convergence and $\left(p_{n k}\right)$-Cesáro summability in the space $F^{2}(E, p, f, s)$ is also made.
\end{abstract}

\section{Introduction \& Motivation}

Ratha and Srivastava 12] and Ghosh and Srivastava [6] introduced and studied generalized classes of composite vector valued single sequence spaces $\mathrm{F}\left(E_{k}, \Lambda\right)$ and $\mathrm{F}\left(E_{k}, f\right)$ respectively, which are defined as

and

$$
F\left(E_{k}, \Lambda\right)=\left\{x=\left(x_{k}\right): x_{k} \in E_{k} \text { for each } k \text { and }\left(g_{E_{k}}\left(v_{k} x_{k}\right)\right) \in F\right\}
$$

$$
F\left(E_{k}, f\right)=\left\{x=\left(x_{k}\right): x_{k} \in E_{k} \text { and the sequence }\left(f\left(g_{E_{k}}\left(x_{k}\right)\right) \in F\right\},\right.
$$

where $\mathrm{F}$ is a normal sequence space with a monotone paranorm $g_{F},\left(E_{k}, g_{E_{k}}\right)$ is Banach space over the field of complex numbers $\mathbf{C}, f$ is a modulus function and $\Lambda(z)=\sum_{k} \frac{z^{k}}{\nu_{k}}$, $\nu=\left(\nu_{k}\right)$ is a sequence of non-zero complex numbers satisfying

$$
\nu=\liminf _{k \rightarrow \infty}\left|\nu_{k}\right|^{\frac{1}{k}}, 0<\nu \leq \infty .
$$

With suitable topologies, the authors have investigated various topological properties for these spaces. The study of these spaces includes many known spaces as particular cases. For example, by specifying $F, E \& f$, one can obtain $w_{0}(f) \& w_{\infty}(f)$ of Maddox [7], $w_{0}(f, p) \& w_{\infty}(f, p)$ of Bilgin [3], $w(f)$ of Öztürk \& Bilgin 11] and others.

To continue the study, we introduce a new space $F^{2}(E, p, f, s)$ of vector valued double sequences which unifies some of the earlier classes on double sequences as particular cases.

Received June 21, 2004; revised May 15, 2007.

2000 Mathematics Subject Classification. 40A05, 40C05, 40 D05.

Key words and phrases. Double sequence, modulus function, statistical convergence, Cesárosummability. 
Also, some important results have been derived on various aspects of double sequences which can be treated as generalization of the results derived by Gökhan et al. ([5] [4]) and Mursaleen et al. [9].

\section{Sequence space $\boldsymbol{F}^{2}(E, p, f, s)$}

Let $\left(\mathrm{E}, q_{E}\right)$ be a seminormed space over the complex field $\mathbb{C}$. Let $S_{2}(E)$ denote the linear space of all double sequences $x=\left(x_{n k}\right)$ with $x_{n k} \in E$ under the usual coordinatewise addition and scalar multiplication. Let $F^{2}$ be a normal scalar double sequence space with monotone paranorm $g_{F^{2}}$ such that co-ordinatewise convergence implies convergence in paranorm $g_{F^{2}}$, i.e.,

$$
a_{n k}^{j, l} \rightarrow 0(j, l \rightarrow \infty) \text { for each } \mathrm{n}, \mathrm{k} \Longrightarrow g_{F^{2}}\left(a^{j, l}\right) \rightarrow 0(j, l \rightarrow \infty)
$$

where $\left(a_{n k}^{j, l}\right)=a^{j, l} \in F^{2}$ for each $j$ and $l \in \mathbb{N}$.

Throughout this chapter, by a convergent double sequence we mean a convergent in Pringsheim's sense. ${ }^{1}$

Let $f$ be a modulus function and $\mathrm{p}=\left(p_{n k}\right)$ be a sequence of strictly positive real numbers and $s \geq 0$. We introduce a new class $F^{2}(E, p, f, s)$ of vector valued double sequences as follows:

$$
\begin{aligned}
F^{2}(E, p, f, s)= & \left\{x=\left(x_{n k}\right) \in S_{2}(E): x_{n k} \in E \text { for each } n, k \in N\right. \text { and the sequence } \\
& \left.\left((n k)^{-s}\left\{f\left(q_{E}\left(x_{n k}\right)\right)\right\}^{p_{n k}}\right) \in F^{2}\right\}
\end{aligned}
$$

Further, we define a topology on $F^{2}(E, p, f, s)$ by a paranorm $g$ which is given by

$$
g(x)=g_{F^{2}}\left[(n k)^{-s}\left\{f\left(q_{E}\left(x_{n k}\right)\right)\right\}^{p_{n k} / M}\right], \text { for } x=\left(x_{n k}\right) \in F^{2}(\mathrm{E}, \mathrm{p}, \mathrm{f}, \mathrm{s})
$$

where $\mathrm{M}=\max (1, H), H=\sup _{n, k} p_{n k}<\infty$ and $\inf p_{n k}>0$.

It is seen that $F^{2}(E, p, f, s)$ turns out to be a complete paranormed space of vector valued double sequences.

It can also be seen that for suitable choice of the sequence space $F^{2}, \mathrm{E}$ and the modulus function $\mathrm{f}$, the space $F^{2}(E, p, f, s)$ includes many of the known scalar as well as vector valued sequence spaces as particular cases.

\section{Application:}

1. If we take $E=\mathbb{C}, f(x)=x, s=0$ and $F^{2}=\ell_{2}^{\infty}$, the space $F^{2}(E, p, f, s)$ gives rise the space $\ell_{2}^{\infty}(p)$ of Gökhan et al. [5].

2. If we take $E=\mathbb{C}, f(x)=x, s=0$ and $F^{2}=c_{2}$, the space $F^{2}(E, p, f, s)$ gives rise the space $c_{2}^{P}(p)$ of Gökhan et al. [4].

\footnotetext{
${ }^{1}$ see section 1.6 of Chapter 1.
} 
3. If we take $E=\mathbb{C}, f(x)=x, s=0$ and $F^{2}=c_{2} \cap \ell_{2}^{\infty}$, the space $F^{2}(E, p, f, s)$ gives rise the space $c_{2}^{P B}(p)$ of Gökhan et al. [4].

4. If we take $E=\mathbb{C}, f(x)=x, s=0, p_{n k} \equiv 1$ and $F^{2}=w^{2}(p), \ell_{2}^{\infty}, c_{2}, c_{0}^{2}$, the space $F^{2}(E, p, f, s)$ gives rise the spaces of Tripathy [13].

\section{Main Results}

Theorem 3.1. $F^{2}(E, p, f, s)$ is a linear space, where $p=\left(p_{n k}\right)$ is a bounded sequence of strictly positive real numbers $\mathcal{B} \inf p_{n k}>0$.

Proof. Let $\mathrm{x}=\left(x_{n k}\right), \mathrm{y}=\left(y_{n k}\right) \in F^{2}(E, p, f, s)$ and $\lambda, \mu \in \mathbb{C}$. Then

$$
\begin{aligned}
(n k)^{-s}\left\{f\left(q_{E}\left(\lambda x_{n k}+\mu y_{n k}\right)\right)\right\}^{p_{n k}} \\
\quad \leq(n k)^{-s}\left\{f\left(q_{E}\left(\lambda x_{n k}\right)+q_{E}\left(\mu y_{n k}\right)\right)\right\}^{p_{n k}} \\
\quad \leq(n k)^{-s}\left\{f\left(q_{E}\left(\lambda x_{n k}\right)\right)+f\left(q_{E}\left(\mu y_{n k}\right)\right)\right\}^{p_{n k}} \\
\quad \leq D(n k)^{-s}\left[\left\{f\left(|\lambda| q_{E}\left(x_{n k}\right)\right)\right\}^{p_{n k}}+\left\{f\left(|\mu| q_{E}\left(y_{n k}\right)\right)\right\}^{p_{n k}}\right] \\
\quad \leq D(1+[|\lambda|])^{H}(n k)^{-s}\left\{f\left(q_{E}\left(x_{n k}\right)\right\}^{p_{n k}}+D(1+[|\mu|])^{H}(n k)^{-s}\left\{f\left(q_{E}\left(y_{n k}\right)\right\}^{p_{n k}}\right.\right.
\end{aligned}
$$

where $\mathrm{D}=\max \left(1,2^{H-1}\right)$. Since $\mathrm{F}^{2}$ is normal, $\lambda x+\mu y \in F^{2}(E, p, f, s)$.

Theorem 3.2. $F^{2}(E, p, f, s)$ is a paranormed space under the paranorm g given by [2.3), where $p=\left(p_{n k}\right)$ is a bounded sequence of strictly positive real numbers 8 $\inf p_{n k}>0$.

Proof. It is clear from the definition of $\mathrm{g}$ that $g(\bar{\theta})=0$ and $g(x)=g(-x)$, where $\bar{\theta}$ is the null element. Again taking $\lambda=1, \mu=1$ in the Theorem 3.1 and the fact that $g_{F^{2}}$ is a monotone paranorm on $F^{2}$, we get $\mathrm{g}(\mathrm{x}+\mathrm{y}) \leq \mathrm{g}(\mathrm{x})+\mathrm{g}(\mathrm{y})$. It is left to prove the continuity of scalar multiplication under $g$.

Suppose $\left\{\lambda_{m}\right\}$ is a sequence of scalars such that $\lambda_{m} \rightarrow \lambda$ as $m \rightarrow \infty$ and let $x^{j, l} \stackrel{g}{\rightarrow}$ $x$ as $j, l \rightarrow \infty$. To show $g\left(\lambda_{m} x^{j, l}-\lambda x\right) \rightarrow 0$ as $j, l \rightarrow \infty$ where $x^{j, l}=\left(x_{n k}^{j, l}\right) \in$ $F^{2}(E, p, f, s)$.

Let

$$
a_{n k}^{j m}=(n k)^{-s}\left\{f\left(\left|\lambda_{m}-\lambda\right| q_{E}\left(x_{n k}\right)\right)\right\}^{p_{n k} / M} .
$$

As $\lambda_{m} \rightarrow \lambda$ as $m \rightarrow \infty$, for sufficiently large $\mathrm{m}$, we can assume that $\left|\lambda_{m}-\lambda\right|<1$. 
Consider

$$
\begin{aligned}
g\left(\lambda_{m} x^{j, l}-\lambda x\right) & =g_{F^{2}}\left[\left((n k)^{-s}\left\{f\left(q_{E}\left[\lambda_{m} x_{n k}^{j, l}-\lambda x_{n k}\right]\right)\right\}^{p_{n k} / M}\right)\right] \\
& \leq g_{F^{2}}\left[\left(( n k ) ^ { - s } \left\{f \left(q _ { E } \left[\left(\lambda_{m}-\lambda\right)\left(x_{n k}^{j, l}-x_{n k}\right)\right.\right.\right.\right.\right. \\
& \left.\left.\left.\left.\left.+\lambda\left(x_{n k}^{j, l}-x_{n k}\right)+\left(\lambda_{m}-\lambda\right) x_{n k}\right]\right)\right\}^{p_{n k} / M}\right)\right] \\
& \leq g_{F^{2}}\left[\left((n k)^{-s}\left\{f\left(q_{E}\left[\left(\lambda_{m}-\lambda\right)\left(x_{n k}^{j, l}-x_{n k}\right)\right]\right)\right\}^{p_{n k} / M}\right)\right] \\
& +g_{F_{2}}\left[(n k)^{-s}\left\{f\left(q_{E}\left[\lambda\left(x_{n k}^{j, l}-x_{n k}\right)\right]\right)\right\}^{p_{n k} / M}\right] \\
& +g_{F^{2}}\left[\left((n k)^{-s}\left\{f\left(q_{E}\left[\left(\lambda_{m}-\lambda\right) x_{n k}\right]\right)\right\}^{p_{n k} / M}\right)\right] \\
& =g_{F^{2}}\left[\left((n k)^{-s}\left\{f\left(\left|\lambda_{m}-\lambda\right| q_{E}\left[x_{n k}^{j, l}-x_{n k}\right]\right)\right\}^{p_{n k} / M}\right)\right] \\
& +g_{F^{2}}\left[\left((n k)^{-s}\left\{f\left(|\lambda| q_{E}\left[x_{n k}^{j, l}-x_{n k}\right]\right)\right\}^{p_{n k} / M}\right)\right] \\
& +g_{F^{2}}\left[\left((n k)^{-s}\left\{f\left(\left|\lambda_{m}-\lambda\right| q_{E}\left[x_{n k}\right]\right)\right\}^{p_{n k} / M}\right)\right] \\
& \equiv \mathrm{I}+\mathrm{II}+\mathrm{III} .
\end{aligned}
$$

Since $\lambda_{m} \rightarrow \lambda$ as $m \rightarrow \infty$, so

$$
\begin{aligned}
\mathrm{I} & \equiv g_{F^{2}}\left[\left((n k)^{-s}\left\{f\left(\left|\lambda_{m}-\lambda\right| q_{E}\left[x_{n k}^{j, l}-x_{n k}\right]\right)\right\}^{p_{n k} / M}\right)\right] \\
& \leq g_{F^{2}}\left[\left((n k)^{-s}\left\{f\left(q_{E}\left[x_{n k}^{j, l}-x_{n k}\right]\right)\right\}^{p_{n k} / M}\right)\right]=g\left(x^{j, l}-x\right) .
\end{aligned}
$$

Again since $f(\lambda)<(1+[|\lambda|]) f(1)$, so as $m \rightarrow \infty$

$$
\begin{aligned}
\mathrm{II} & \equiv g_{F^{2}}\left[\left((n k)^{-s}\left\{f\left(|\lambda| q_{E}\left[x_{n k}^{j, l}-x_{n k}\right]\right)\right\}^{p_{n k} / M}\right)\right] \\
& \leq g_{F^{2}}\left[\left((n k)^{-s}\left\{(1+[|\lambda|]) f\left(q_{E}\left[x_{n k}^{j, l}-x_{n k}\right]\right)\right\}^{p_{n k} / M}\right)\right] \\
& =(1+[|\lambda|]) g\left(x^{j, l}-x\right) .
\end{aligned}
$$

Hence from (3.2) using (3.1) we get

$$
\begin{aligned}
& g\left(\lambda_{m} x^{j, l}-\lambda x\right) \\
= & g\left(x^{j, l}-x\right)+(1+[|\lambda|]) g\left(x^{j, l}-x\right)+\left[g_{F^{2}}\left(a_{n k}^{m}\right)\right]
\end{aligned}
$$

Also, since

$$
f\left(\left|\lambda_{m}-\lambda\right| q_{E}\left(x_{n k}\right)\right)<f\left(q_{E}\left(x_{n k}\right)\right)
$$


holds because $\left|\lambda_{m}-\lambda\right|<1$ for sufficiently large $\mathrm{m}$ and $F^{2}$ is normal so $a^{m}=\left(a_{n k}^{m}\right) \in F^{2}$ for sufficiently large $\mathrm{m}$. Obviously for each $\mathrm{n}, \mathrm{k}, a_{n k}^{m} \rightarrow 0$ as $m \rightarrow \infty$. So by the condition (2.1), we get $g_{F^{2}}\left(a_{n k}^{m}\right) \rightarrow 0$ for sufficiently large m. Again II and III tend to zero as $j, l \rightarrow \infty \& m \rightarrow \infty$, because $\lambda_{m} \rightarrow \lambda \& x^{j, l} \stackrel{g}{\rightarrow} x$. Hence we get

$$
g\left(\lambda_{m} x^{j, l}-\lambda x\right) \rightarrow 0 \text { as } m \rightarrow \infty \text { and } j, l \rightarrow \infty .
$$

Hence the proof.

Theorem 3.3. $F^{2}(\mathrm{E}, \mathrm{p}, \mathrm{f}, \mathrm{s})$ is a $K_{2}$-space if $F^{2}$ is a $K_{2}$-space.

Proof. Define $P_{n k}: F^{2}(E, p, f, s) \rightarrow E$ as $P_{n k}(x)=x_{n k}, n, k=1,2,3, \ldots$, where $x=\left(x_{n k}\right) \in F^{2}(E, p, f, s)$. To show $P_{n k}$ is continuous.

Let $\left(x^{j, l}\right)=\left(\left(x_{n k}^{j, l}\right)\right)$ be a sequence in $F^{2}(\mathrm{E}, \mathrm{p}, \mathrm{f}, \mathrm{s})$ such that

$$
g\left(x^{j, l}\right) \rightarrow 0 \text { as } j, l \rightarrow \infty .
$$

Since $F^{2}$ is a $K_{2}$-space, $g\left(x^{j, l}\right) \rightarrow 0$ as $j, l \rightarrow \infty$ implies that

$$
(n k)^{-s}\left\{f\left(q_{E}\left(x_{n k}^{j, l}\right)\right)\right\}^{p_{n k} / M} \rightarrow 0 \text { as } j, l \rightarrow \infty, \text { for each } \mathrm{n}, \mathrm{k} .
$$

We claim that $q_{E}\left(x_{n k}^{j, l}\right) \rightarrow 0$ as $j, l \rightarrow \infty$, because $f$ is continuous and increasing. This implies

$$
q_{E}\left(P_{n k}\left(x^{j, l}\right)\right)=q_{E}\left(x_{n k}^{j, l}\right) \rightarrow 0 \text { for } j, l \rightarrow \infty .
$$

Hence the proof.

Theorem 3.4. $F^{2}(E, p, f, s)$ is a normal space.

Proof. The proof is straightforward, so we omit it.

Theorem 3.5. $F^{2}(E, p, f, s)$ is complete with respect to the paranorm $g$ if $\left(E, q_{E}\right)$ is complete, and $F^{2}$ is normal $K_{2}$-space, where $\left(p_{n k}\right)$ is bounded sequence of strictly positive real numbers such that inf $p_{n k}>0$.

Proof. Let $x^{j, l}=\left(x_{n k}^{j, l}\right)$ be a Cauchy sequence in $F^{2}(\mathrm{E}, \mathrm{p}, \mathrm{f}, \mathrm{s})$. So

$$
g\left(x^{j, l}-x^{r, t}\right) \rightarrow 0 \text { as } j, l, r, t \rightarrow \infty
$$

i.e.,

$$
g_{F_{2}}\left[\left((n k)^{-s}\left\{f\left(q_{E}\left(x_{n k}^{j, l}-x_{n k}^{r, t}\right)\right)\right\}^{p_{n k} / M}\right)\right] \rightarrow 0 \text { as } j, l, r, t \rightarrow \infty
$$

Since $F_{2}(\mathrm{E}, \mathrm{p}, \mathrm{f}, \mathrm{s})$ is a $K_{2}$ space and $f$ is continuous, so for each n,k

$$
(n k)^{-s / M} q_{E}\left(x_{n k}^{j, l}-x_{n k}^{r, t}\right) \rightarrow 0 \text { as } j, l, r, t \rightarrow \infty .
$$


Hence for fixed $\mathrm{n}, \mathrm{k}$

$$
q_{E}\left(x_{n k}^{j, l}-x_{n k}^{r, t}\right) \rightarrow 0 \text { as } j, l, r, t \rightarrow \infty .
$$

This implies that for fixed $\mathrm{n}, \mathrm{k},\left(x_{n k}^{j, l}\right)$ behaves as a Cauchy sequence in E. But $\left(E, q_{E}\right)$ is complete, so there exist $x=\left(x_{n k}\right) \in \mathrm{E}$ such that

$$
q_{E}\left(x_{n k}^{j, l}-x_{n k}\right) \rightarrow 0 \text { as } j, l \rightarrow \infty .
$$

So

$$
a_{n k}^{j, l} \rightarrow 0(\text { as } j, l \rightarrow \infty) \text { for each } n, k \text { (since } f \text { is continuous) }
$$

where

$$
a_{n k}^{j, l}=(n k)^{-s}\left\{f\left(q_{E}\left(x_{n k}^{j, l}-x_{n k}\right)\right)\right\}^{p_{n k} / M}, n, k=1,2, \ldots
$$

Since for each n, $\mathrm{k}, a_{n k}^{j, l} \rightarrow 0$ as $j, l \rightarrow \infty$, so choose $\delta_{n k}^{j, l}$ such that

$$
a_{n k}^{j, l} \leq \delta_{n k}^{j, l}(n k)^{-s}\left\{f\left(q_{E}\left(x_{n k}^{j, l}\right)\right)\right\}^{p_{n k} / M} \text { where } 0<\delta_{n k}^{j, l}<1 .
$$

Clearly $a^{j, l} \in F^{2}$, for each $\mathrm{m}$, because $F^{2}$ is normal.

Hence,

$$
g_{F_{2}}\left[\left((n k)^{-s}\left\{f\left(q_{E}\left(x_{n k}^{j, l}-x_{n k}\right)\right)\right\}^{p_{n k} / M}\right)\right] \rightarrow 0 \text { as } j, l \rightarrow \infty
$$

i.e., $g\left(x^{j, l}-x\right) \rightarrow 0$ as $j, l \rightarrow \infty$.

Now using (3.3) and (3.4) we get

$$
(n k)^{-s}\left\{f\left(q_{E}\left(x_{n k}\right)\right)\right\}^{p_{n k} / M} \leq\left(\delta_{n k}^{j, l}+1\right)(n k)^{-s}\left\{f\left(q_{E}\left(x_{n k}^{j, l}\right)\right)\right\}^{p_{n k} / M} .
$$

Since $F^{2}$ is normal space and $x^{j, l} \in F^{2}(\mathrm{E}, \mathrm{p}, \mathrm{f}, \mathrm{s}), x=\left(x_{n k}\right) \in F^{2}(\mathrm{E}, \mathrm{p}, \mathrm{f}, \mathrm{s})$. Hence the proof.

Theorem 3.6. Let $f$ be a modulus function such that $f(u v)=f(u) f(v)$ where $u$, $v$ are positive scalars. Let $E$ be a commutative normal sequence algebra under $\circ$, where $x_{n k} \circ y_{n k}=x_{n k} y_{n k}$ and $F^{2}$ is a normal sequence algebra under the multiplication $\circ^{\prime}$, defined as $\left(a_{n k}\right) \circ^{\prime}\left(b_{n k}\right)=\left(a_{n k} b_{n k}\right)$, where $\left(a_{n k}\right),\left(b_{n k}\right) \in F^{2}$. Then $F^{2}(E, p, f, s)$ is a commutative sequence algebra.

Proof. Let $x=\left(x_{n k}\right)$ and $y=\left(y_{n k}\right) \in F^{2}(E, p, f, s)$. Consider

$$
\begin{aligned}
(n k & )^{-2 s}\left\{f\left(q_{E}\left(x_{n k} \circ y_{n k}\right)\right)\right\}^{p_{n k}} \\
& =(n k)^{-2 s}\left\{f\left(q_{E}\left(x_{n k} y_{n k}\right)\right)\right\}^{p_{n k}} \\
& \leq(n k)^{-2 s}\left\{f\left(q_{E}\left(x_{n k}\right) q_{E}\left(y_{n k}\right)\right)\right\}^{p_{n k}} \text { (since E is a normed algebra) } \\
& =(n k)^{-2 s}\left\{f\left(q_{E}\left(x_{n k}\right)\right) f\left(q_{E}\left(y_{n k}\right)\right)\right\}^{p_{n k}} \text { (by given condition) } \\
& =(n k)^{-s}\left\{f\left(q_{E}\left(x_{n k}\right)\right)\right\}^{p_{n k}}(n k)^{-s}\left\{f\left(q_{E}\left(y_{n k}\right)\right)\right\}^{p_{n k}} \in F^{2}
\end{aligned}
$$


as $x, y \in F^{2}(E, p, f, s)$, which implies $\left(x_{n k} y_{n k}\right) \in F^{2}(E, p, f, s)$. So $F^{2}(E, p, f, s)$ is a sequence algebra. Further, it can be seen easily that $F^{2}(E, p, f, s)$ is a commutative sequence algebra as $E$ is commutative.

Remark 3.1. The condition inf $p_{n k}>0$ is not required when $F^{2} \equiv c_{0}^{2}, c^{2}, \ell_{p}^{2}, p \geq 1$. But for $F^{2} \equiv \ell_{\infty}^{2}$ the condition inf $p_{n k}>0$ is required. For the sake of completeness we have chosen this condition in general.

Now, we prove the following lemma which will be used in sequel:

Lemma 3.1. Let $f_{1}, f_{2}$ be modulus functions and $0<\delta<1$. Let $f_{1}(t)>\delta$ for $t \geq 0$, then

$$
\left(f_{2} \circ f_{1}\right)(t) \leq \frac{2 f_{2}(1)}{\delta} f_{1}(t)
$$

Proof. Since for $f_{1}(t)>\delta$,

$$
f_{1}(t)<\frac{f_{1}(t)}{\delta}<1+\left[\frac{f_{1}(t)}{\delta}\right]
$$

we have

$$
\left(f_{2} \circ f_{1}\right)(t) \leq\left(1+\left[\frac{f_{1}(t)}{\delta}\right]\right) f_{2}(1) \leq 2 \frac{f_{1}(t)}{\delta} f_{2}(1) .
$$

Some inclusion relations which are known for single sequence spaces are extended analogously to double sequence spaces as follows:

Theorem 3.7. Let $F^{2}$ be a normal sequence space. Then the following inequalities hold:

1. If $\sup _{t>0} \frac{f_{1}(t)}{f_{2}(t)}<\infty$, then $F^{2}\left(E, p, f_{2}, s\right) \subseteq F^{2}\left(E, p, f_{1}, s\right)$.

2. $F^{2}\left(E, p, f_{1}, s\right) \cap F^{2}\left(E, p, f_{2}, s\right) \subseteq F^{2}\left(E, p, f_{1}+f_{2}, s\right)$.

3. $F^{2}\left(E, p, f_{1}, s\right) \subseteq F^{2}\left(E, p, f_{2} \circ f_{1}, s\right)$ if $(n k)^{-s} \in F^{2}$, where $\left(f_{2} \circ f_{1}\right)(t)=f_{2}\left(f_{1}(t)\right)$ and $\inf p_{n k}>0 \mathscr{E} \sup p_{n k}<\infty$.

4. If $s_{1} \leq s_{2}$, then $F^{2}\left(E, p, f_{1}, s_{1}\right) \subseteq F^{2}\left(E, p, f_{1}, s_{2}\right)$.

Proof (i). Let $x=\left(x_{n k}\right) \in F^{2}\left(E, p, f_{2}, s\right)$.

Since $\sup _{t>0} \frac{f_{1}(t)}{f_{2}(t)}<\infty$ is given, $\exists L>0$ such that $f_{1}(t) \leq L f_{2}(t)$ for all $\mathrm{t}>0$ and hence

$(n k)^{-s}\left\{f_{1}\left(q_{E}\left(x_{n k}\right)\right)\right\}^{p_{n k}} \leq \max \left(1, L^{H}\right)(n k)^{-s}\left\{f_{2}\left(q_{E}\left(x_{n k}\right)\right)\right\}^{p_{n k}}$ for each $\mathrm{n}$ and $\mathrm{k}$.

Since $F^{2}$ is normal, so the result follows. 
Proof (ii). Let $x=\left(x_{n k}\right) \in F^{2}\left(E, p, f_{1}, s\right) \cap F^{2}\left(E, p, f_{2}, s\right)$. Consider

$$
\begin{aligned}
(n k)^{-s}\left\{\left(f_{1}+f_{2}\right)\left(q_{E}\left(x_{n k}\right)\right)\right\}^{p_{n k}} & =(n k)^{-s}\left[\left\{f_{1}\left(q_{E}\left(x_{n k}\right)\right)\right\}+\left\{f_{2}\left(q_{E}\left(x_{n k}\right)\right)\right\}\right]^{p_{n k}} \\
& \leq D(n k)^{-s}\left[\left\{f_{1}\left(q_{E}\left(x_{n k}\right)\right)\right\}^{p_{n k}}+\left\{f_{2}\left(q_{E}\left(x_{n k}\right)\right)\right\}^{p_{n k}}\right]
\end{aligned}
$$

for each $n$ and $k$ and $\mathrm{D}=\max \left(1,2^{H-1}\right)$. Result follows as $F^{2}$ is normal sequence space.

Proof (iii). Let us choose $\delta$ such that $0<\delta<1$. Let

$$
\begin{gathered}
N_{1}=\left\{(n, k) \in N \times N: f_{1}\left(q_{E}\left(x_{n k}\right)\right) \leq \delta\right\} \\
N_{2}=\left\{(n, k) \in N \times N: f_{1}\left(q_{E}\left(x_{n k}\right)\right)>\delta\right\} .
\end{gathered}
$$

If $(\mathrm{n}, \mathrm{k}) \in N_{1}$, then

$$
\left(f_{2} \circ f_{1}\right)\left(q_{E}\left(x_{n k}\right)\right) \leq f_{2}(\delta)
$$

Hence

$$
(n k)^{-s}\left(\left(f_{2} \circ f_{1}\right)\left(q_{E}\left(x_{n k}\right)\right)\right)^{p_{n k}} \leq \eta_{1}(n k)^{-s},
$$

where

$$
\eta_{1}=\max \left[\left\{f_{2}(\delta)\right\}^{\inf p_{n k}},\left\{f_{2}(\delta)\right\}^{\sup p_{n k}}\right] .
$$

Again for $(\mathrm{n}, \mathrm{k}) \in N_{2}$,

$$
\begin{aligned}
(n k)^{-s}\left(\left(f_{2} \circ f_{1}\right)\left(q_{E}\left(x_{n k}\right)\right)\right)^{p_{n k}} & \leq(n k)^{-s}\left[\frac{2 f_{2}(1)}{\delta} f_{1}\left(q_{E}\left(x_{n k}\right)\right)\right]^{p_{n k}} \quad(\text { by Lemma 3.1) } \\
& \leq \eta_{2}(n k)^{-s}\left[f_{1}\left(q_{E}\left(x_{n k}\right)\right)\right]^{p_{n k}}
\end{aligned}
$$

where

$$
\eta_{2}=\max \left\{\left\{\frac{2 f_{2}(1)}{\delta}\right\}^{\inf p_{n k}},\left\{\frac{2 f_{2}(1)}{\delta}\right\}^{\sup p_{n k}}\right\}
$$

Let $\eta=\max \left(\eta_{1}, \eta_{2}\right)$.

From (3.5) and (3.6) wet for $(n, k) \in N_{1} \cup N_{2}$,

$$
(n k)^{-s}\left(\left(f_{2} \circ f_{1}\right)\left(q_{E}\left(x_{n k}\right)\right)\right)^{p_{n k}} \leq \eta\left[(n k)^{-s}+(n k)^{-s}\left[f_{1}\left(q_{E}\left(x_{n k}\right)\right)\right]^{p_{n k}}\right] .
$$

Since $\left((n k)^{-s}\right) \in F^{2}$ and $F^{2}(\mathrm{E}, \mathrm{f}, \mathrm{p}, \mathrm{s})$ is normal, so the result follows.

Proof (iv). For $s_{1} \leq s_{2}$

$$
(n k)^{-s_{2}}\left[f\left(q_{E}\left(x_{n k}\right)\right)\right]^{p_{n k}} \leq(n k)^{-s_{1}}\left[f\left(q_{E}\left(x_{n k}\right)\right)\right]^{p_{n k}} \text { for every } \mathrm{n}, \mathrm{k} .
$$


By using the normality of $F^{2}$, the result is obtained.

\section{Multiplier set of $F^{2}(E, p, f, s)$}

This section deals with some inclusion relations between the set $F^{2}(E, p, f, s)$ and its multiplier set.

We define multiplier set of $F^{2}(E, p, f, s)$ as

$$
\begin{aligned}
M^{2} & {\left[F^{2}(E, p, f, s)\right] } \\
& =\left\{a=\left(a_{n k}\right) \in E:\left(a_{n k} x_{n k}\right) \in F^{2}(E, p, f, s) \text { for all } x=\left(x_{n k}\right) \in F^{2}(E, p, f, s)\right\}
\end{aligned}
$$

where $E$ is taken as normed algebra. Now, we prove the following theorems:

Theorem 4.1. Let $E$ be normed algebra and $F^{2}$ be a normal sequence space. Then

$$
\ell_{2}^{\infty}(E) \subseteq M^{2}\left[F^{2}(E, p, f, s)\right]
$$

where

$$
\ell_{2}^{\infty}(E)=\left\{a=\left(a_{n k}\right): a_{n k} \in E \text { and } \sup _{\mathrm{n}, \mathrm{k}} \mathrm{q}_{\mathrm{E}}\left(\mathrm{a}_{\mathrm{nk}}\right)<\infty\right\}
$$

Proof. Let $a=\left(a_{n k}\right) \in \ell_{2}^{\infty}(E)$ and $x=\left(x_{n k}\right) \in F^{2}(E, p, f, s)$.

Let $\mathrm{B}=\sup _{n, k} q_{E}\left(a_{n k}\right)<\infty$. Now,

$$
\begin{aligned}
(n k)^{-s}\left\{f\left(q_{E}\left(a_{n k} x_{n k}\right)\right)\right\}^{p_{n k}} \\
\quad \leq(n k)^{-s}\left\{f\left(q_{E}\left(a_{n k}\right) q_{E}\left(x_{n k}\right)\right)\right\}^{p_{n k}} \text { (since E is normed algebra) } \\
\quad<(1+[B])^{H}(n k)^{-s}\left\{f\left(q_{E}\left(x_{n k}\right)\right)\right\}^{p_{n k}}
\end{aligned}
$$

where $\left[B^{H}\right]$ denotes the integral part of $B^{H}$.

Since $\mathrm{F}^{2}$ is normal, this implies $\left(a_{n k} x_{n k}\right) \in F^{2}(E, p, f, s)$ and consequently $\left(a_{n k}\right) \in$ $M^{2}\left[F^{2}(E, p, f, s)\right]$. Hence the proof.

Theorem 4.2. For any modulus function satisfying $f(\alpha \beta) \leq f(\alpha)+f(\beta), \alpha, \beta \in$ $[0, \infty)$

$$
F^{2}(E, p, f, s) \subseteq M^{2}\left[F^{2}(E, p, f, s)\right],
$$

where $E$ is a normed algebra.

Proof. Let $x=\left(x_{n k}\right) \in F^{2}(E, p, f, s)$. We want to show that $x=\left(x_{n k}\right) \in M^{2}\left[F^{2}(E\right.$, $p, f, s)]$, i.e., to show $\left(x_{n k} y_{n k}\right) \in F^{2}(E, p, f, s)$ for all $y=\left(y_{n k}\right) \in F^{2}(E, p, f, s)$.

Consider

$$
q_{E}\left(x_{n k} y_{n k}\right) \leq q_{E}\left(x_{n k}\right) q_{E}\left(y_{n k}\right)
$$

since $E$ is a normed algebra. 
Then

$$
f\left(q_{E}\left(x_{n k} y_{n k}\right)\right) \leq f\left(q_{E}\left(x_{n k}\right) q_{E}\left(y_{n k}\right)\right) \leq f\left(q_{E}\left(x_{n k}\right)\right)+f\left(q_{E}\left(y_{n k}\right)\right) .
$$

Thus

$$
\begin{aligned}
(n k)^{-s}\left\{f\left(q_{E}\left(x_{n k} y_{n k}\right)\right)\right\}^{p_{n k}} & \leq(n k)^{-s}\left\{f\left(q_{E}\left(x_{n k}\right)\right)+f\left(q_{E}\left(y_{n k}\right)\right)\right\}^{p_{n k}} \\
& \leq D(n k)^{-s}\left[\left\{f\left(q_{E}\left(x_{n k}\right)\right)\right\}^{p_{n k}}+\left\{f\left(q_{E}\left(y_{n k}\right)\right)\right\}^{p_{n k}}\right]
\end{aligned}
$$

where $\mathrm{D}=\max \left(1,2^{H-1}\right)$.

This implies $x y \in F^{2}(E, p, f, s)$ and hence $x \in M^{2}\left[F^{2}(E, p, f, s)\right]$.

\section{Ideals of $F^{2}(E, p, f, s)$}

Let $\mathrm{I}^{2}$ be a normal subspace of $F^{2}$, where $F^{2}$ is a sequence algebra. Let $E$ be commutative normed algebra and $\mathrm{S}_{2}(E)$ is the linear space of all sequences $x=\left(x_{n k}\right)$ with $x_{n k} \in E$ under the usual coordinatewise addition and scalar multiplication.

$$
\begin{aligned}
& I^{2}(E, p, f, s) \\
& \quad=\left\{x=\left(x_{n k}\right): x_{n k} \in S(E) \text { for each n,k and }\left((n k)^{-s}\left\{f\left(q_{E}\left(x_{n k}\right)\right)\right\}^{p_{n k}}\right) \in I^{2}\right\}
\end{aligned}
$$

It is easy to check that $I^{2}(E, p, f, s)$ is a subspace of $F^{2}(E, p, f, s)$.

Theorem 5.1. If $I^{2}$ is closed subspace of $F^{2}$ and $F^{2}$ is a normal $K_{2}$-space then for $0<p_{n k} \leq 1, I^{2}(E, p, f, s)$ is a closed subspace of $F^{2}(E, p, f, s)$.

Proof. It is easy to show that $I^{2}(E, p, f, s)$ is a subspace of $F^{2}(E, p, f, s)$. Next, to show it is closed, we take $x=\left(x_{n k}\right) \in \overline{I^{2}(E, p, f, s)}$, the closure of $I^{2}(E, p, f, s)$. This implies the existence of a sequence $x^{j, l}=\left(\left(x_{n k}^{j, l}\right)\right) \in I^{2}(E, p, f, s)$ such that

$$
g\left(x^{j, l}-x\right) \rightarrow 0 \text { as } j, l \rightarrow \infty
$$

for some $x=\left(x_{n k}\right) \in F^{2}(E, p, f, s)$.

Consequently,

$$
\begin{array}{r}
g_{F^{2}}\left[\left((n k)^{-s}\left\{f\left(q_{E}\left(x_{n k}^{j, l}-x_{n k}\right)\right)\right\}^{p_{n k}}\right)\right] \rightarrow 0 \text { as } m \rightarrow \infty . \\
\left(\operatorname{since} \mathrm{M}=\max \left(1, \sup p_{n k}\right)=1\right)
\end{array}
$$

Since $F^{2}$ is $K_{2}$-space and $f$ is continuous at 0 , so,

$$
q_{E}\left(x_{n k}^{j, l}-x_{n k}\right) \rightarrow 0 \text { as } j, l \rightarrow \infty \text { for each } \mathrm{n}, \mathrm{k} .
$$


Consider

$$
\begin{aligned}
\left\{f\left(q_{E}\left(x_{n k}^{j, l}\right)\right)\right\}^{p_{n k}} & \leq\left\{f\left(q_{E}\left(x_{n k}^{j, l}-x_{n k}+x_{n k}\right)\right)\right\}^{p_{n k}} \\
& \leq\left\{f\left(q_{E}\left(x_{n k}^{j, l}-x_{n k}\right)\right)+f\left(q_{E}\left(x_{n k}\right)\right)\right\}^{p_{n k}} \\
& \leq\left\{f\left(q_{E}\left(x_{n k}^{j, l}-x_{n k}\right)\right)\right\}^{p_{n k}}+\left\{f\left(q_{E}\left(x_{n k}\right)\right)\right\}^{p_{n k}}\left(\text { as } 0<p_{n k} \leq 1\right)
\end{aligned}
$$

Therefore

$$
\left\{f\left(q_{E}\left(x_{n k}^{j, l}\right)\right)\right\}^{p_{n k}}-\left\{f\left(q_{E}\left(x_{n k}\right)\right)\right\}^{p_{n k}} \leq\left\{f\left(q_{E}\left(x_{n k}^{j, l}-x_{n k}\right)\right)\right\}^{p_{n k}} .
$$

Since $F^{2}$ is normal and $g_{F^{2}}$ is a monotone paranorm, so (5.2) implies that

$$
\left((n k)^{-s}\left(\left\{f\left(q_{E}\left(x_{n k}^{j, l}\right)\right)\right\}^{p_{n k}}-\left\{f\left(q_{E}\left(x_{n k}\right)\right)\right\}^{p_{n k}}\right)\right) \in F^{2} .
$$

So we get from (5.2) and (5.3)

$$
\begin{aligned}
& g_{F^{2}}\left[(n k)^{-s}\left(\left\{f\left(q_{E}\left(x_{n k}^{j, l}\right)\right)\right\}^{p_{n k}}-\left\{f\left(q_{E}\left(x_{n k}\right)\right)\right\}^{p_{n k}}\right)\right] \\
\leq & g_{F^{2}}\left[(n k)^{-s}\left\{f\left(q_{E}\left(x_{n k}^{j, l}-x_{n k}\right)\right)\right\}^{p_{n k}}\right] \\
= & g\left(x^{j, l}-x\right)
\end{aligned}
$$

Using (5.1) we get,

$$
g_{F^{2}}\left[(n k)^{-s}\left(\left\{f\left(q_{E}\left(x_{n k}^{j, l}\right)\right)\right\}^{p_{n k}}\right)-(n k)^{-s}\left(\left\{f\left(q_{E}\left(x_{n k}\right)\right)\right\}^{p_{n k}}\right)\right] \rightarrow 0 \text { as } m \rightarrow \infty .
$$

Since $I^{2}$ is closed in $F^{2}$, it is clear from (5.4) that,

$$
\left((n k)^{-s}\left\{f\left(q_{E}\left(x_{n k}\right)\right)\right\}^{p_{n k}}\right) \in I^{2} .
$$

Hence $x=\left(x_{n k}\right) \in I^{2}(E, p, f, s)$.

Theorem 5.2. Let $I^{2}$ be an ideal of $F^{2}$. Further, let the modulus function $f$ satisfies $f(u v)=f(u) f(v)$ where $u, v$ are scalars. Then $I^{2}(E, p, f, s)$ is an ideal of $F^{2}(E, p, f, s)$.

Proof. For $x=\left(x_{n k}\right) \in I^{2}(E, p, f, s)$ and $r=\left(r_{n k}\right) \in F^{2}(E, f, p, s)$,

$$
\begin{aligned}
(n k)^{-2 s}\left\{f\left(q_{E}\left(r_{n k} x_{n k}\right)\right)\right\}^{p_{n k}} & \leq(n k)^{-2 s}\left\{f\left(q_{E}\left(r_{n k}\right) q_{E}\left(x_{n k}\right)\right)\right\}^{p_{n k}} \\
& =(n k)^{-s}\left\{f\left(q_{E}\left(r_{n k}\right)\right)\right\}^{p_{n k}}(n k)^{-s}\left\{f\left(q_{E}\left(x_{n k}\right)\right)\right\}^{p_{n k}} \in I^{2}
\end{aligned}
$$

As because $I^{2}$ is an ideal of $F^{2}$,

$$
\left((n k)^{-s}\left\{f\left(q_{E}\left(r_{n k}\right)\right)\right\}^{p_{n k}}(n k)^{-s}\left\{f\left(q_{E}\left(x_{n k}\right)\right)\right\}^{p_{n k}}\right) \in I^{2} .
$$


Further normality of $I^{2}$ implies

$$
\left((n k)^{-2 s}\left\{f\left(q_{E}\left(r_{n k} x_{n k}\right)\right)\right\}^{p_{n k}}\right) \in I^{2}
$$

and hence $r x \in I^{2}(E, p, f, s)$.

Similarly it can be shown that $x r \in I^{2}(E, f, s)$ which completes the proof.

Theorem 5.3. If $I^{2}$ is a subspace of $\ell_{2}^{\infty}$, for any unbounded function $f, I^{2}(E, p, f, s)$ is an ideal of $\ell_{2}^{\infty}(E, p, f, s)$.

Proof. Let $x=\left(x_{n k}\right) \in I^{2}(E, p, f, s)$ and $\ell=\left(\ell_{n k}\right) \in \ell_{2}^{\infty}(E, p, f, s)$. So

$$
\sup _{n, k}(n k)^{-s}\left\{f\left(q_{E}\left(\ell_{n k}\right)\right)\right\}^{p_{n k}}<\infty
$$

But $f$ is unbounded and in order to hold (5.5), it follows that $\ell=\left(\ell_{n k}\right) \in \ell_{2}^{\infty}(E)$.

Let

$$
T=\sup _{n, k} q_{E}\left(\ell_{n k}\right)
$$

Then

$$
\begin{aligned}
(n k)^{-s}\left\{f\left(q_{E}\left(\ell_{n k} x_{n k}\right)\right)\right\}^{p_{n k}} & \leq(n k)^{-s}\left\{f\left(q_{E}\left(\ell_{n k}\right) q_{E}\left(x_{n k}\right)\right)\right\}^{p_{n k}} \\
& \leq(n k)^{-s}\left\{f\left(T q_{E}\left(x_{n k}\right)\right)\right\}^{p_{n k}} \\
& \leq(1+[T])^{H}(n k)^{-s}\left\{f\left(q_{E}\left(x_{n k}\right)\right)\right\}^{p_{n k}}
\end{aligned}
$$

Hence by the normality of $I^{2}$, it follows that $\ell x \in I^{2}(E, p, f, s)$.

Similarly we can show that $x \ell \in I^{2}(E, p, f, s)$.

\section{Statistical convergence and strongly $\left(p_{n k}\right)$-Cesáro summability}

The concepts of Cesáro summability and strongly p-Cesáro summability for double sequences are introduced by Moricz [8] while the notion of statistical convergence for double sequences has been discussed by Mursaleen et al. 9].

Mursaleen et al. 9] first introduced and extended the concept of statistical convergence for double sequences of real or complex numbers after defining the analogue concept of natural density for double sequences as follows:

Let $\mathrm{K} \subseteq \mathbb{N} \times \mathbb{N}$ be a two dimensional set of positive integers and let

$$
K(n, m)=C(\{(i, j): i \leq n \text { and } j \leq m\}) .
$$

where $\mathrm{C}(\mathrm{A})$ denotes the cardinality of the set $\mathrm{A}$. 
If the sequence $\left(\frac{K(n, m)}{n m}\right)$ has a limit in Pringsheim's sense [1], then we say that $\mathrm{K}$ has double natural density $\delta_{2}(K)$ and is written as

$$
\delta_{2}(K)=P-\lim _{n, m} \frac{K(n, m)}{n m}
$$

Mursaleen et al. 9] defined analogously the statistical convergence and statistical Cauchy convergence for double sequences $x=\left(x_{n k}\right)$ as follows:

Definition 6.1. A real double sequence $x=\left(x_{i j}\right)$ is said to be statistically convergent to the number $\ell$, if for each $\epsilon>0$, the set

$$
\left\{(i, j): i \leq n \text { and } \mathrm{j} \leq \mathrm{m}:\left|\mathrm{x}_{\mathrm{ij}}-\ell\right| \geq \epsilon\right\}
$$

has double natural density zero in the Pringsheim's sense [1], i.e.,

$$
P-\lim _{m, n} \frac{1}{m n} C\left(\left\{(i, j): i \leq n \& j \leq m,\left|x_{i j}-\ell\right| \geq \epsilon\right\}\right)=0
$$

and this is denoted as $\mathrm{st}_{2}-\lim _{i, j} x_{i j}=\ell$. We denote the set of all statistically convergent sequences (in Pringsheim's sense) by $s t_{2}$.

Definition 6.2. A real double sequence $x=\left(x_{i j}\right)$ is said to be statistically Cauchy, if for each $\epsilon>0$ there exist $A=A(\epsilon)$ and $B=B(\epsilon)$ such that for all $\mathrm{i}, \mathrm{p} \geq \mathrm{A}, \mathrm{j}, \mathrm{q} \geq \mathrm{B}$, the set

$$
\left\{(i, j): i \leq r \text { and } \mathrm{j} \leq \mathrm{s}:\left|\mathrm{x}_{\mathrm{ij}}-\mathrm{x}_{\mathrm{pq}}\right| \geq \epsilon\right\}
$$

has double natural density zero in Pringsheim's sense.

In this section, we have extended the concepts of statistical convergence and Cesárosummability to the generalized vector valued double sequence space $F^{2}(E, p, f, s)$ as follows:

Definition 6.3. A vector valued double sequence $\mathrm{x}=\left(x_{i j}\right) \in F^{2}(E, p, f, s)$ is said to be statistically convergent to $\mathrm{L}$ if for each $\epsilon>0$, the set

$$
\left\{(i, j), i \leq n, j \leq k:(i j)^{-s}\left\{f\left(q_{E}\left(x_{i j}-L\right)\right)\right\}^{p_{i j}} \geq \epsilon\right\}
$$

has double natural density zero.

In this case we write $x_{i j} \stackrel{s^{2}}{\longrightarrow(E, p, f, s)} L$. It is easy to check that $\mathrm{L}$ is unique.

Definition 6.4. Let $x=\left(x_{i j}\right)$ be a vector valued double sequence in $F^{2}(\mathrm{E}, \mathrm{p}, \mathrm{f}, \mathrm{s})$ and $p=\left(p_{i j}\right)$ be a sequence of strictly positive real numbers. Then $x=\left(x_{i j}\right)$ is said to be strongly $\left(p_{i j}\right)$-Cesáro-type summable to $\ell$ if

$$
\lim _{n, k \rightarrow \infty} \frac{1}{n k} \sum_{i=1}^{n} \sum_{j=1}^{k}\left[(i j)^{-s}\left\{f\left(q_{E}\left(x_{i j}-\ell\right)\right\}^{p_{i j}}\right]=0 .\right.
$$


Note 6.1. Let $s_{E, p, f, s}^{2}, w_{E, p, f, s}^{2}$ denote the space of all statistically convergent vector valued double sequences and the space of all strongly $\left(p_{i j}\right)$-Cesáro-type summable vector valued double sequences respectively.

Theorem 6.1. A vector valued double sequence $x=\left(x_{i j}\right) \in F^{2}(E, p, f, s)$ is statistically convergent to $\ell$ if it is strongly $\left(p_{i j}\right)$-Cesáro-type suummable to $\ell$.

Proof. Let

$$
I_{1}(\epsilon)=\left\{(i, j), i \leq n, j \leq k:\left[(i j)^{-s}\left\{f\left(q_{E}\left(x_{i j}-\ell\right)\right)\right\}^{p_{i j}}\right] \geq \epsilon\right\} .
$$

Let us assume that $x=\left(x_{i j}\right)$ is strongly $\left(p_{i j}\right)$-Cesáro suummable to $\ell$. Then

$$
\begin{aligned}
& \frac{1}{n k} \sum_{i=1}^{n} \sum_{j=1}^{k}\left[(i j)^{-s}\left\{f\left(q_{E}\left(x_{i j}-\ell\right)\right)\right\}^{p_{i j}}\right] \\
& \quad=\frac{1}{n k}\left[\sum_{(i, j) \in I_{1}}\left[(i j)^{-s}\left\{f\left(q_{E}\left(x_{i j}-\ell\right)\right)\right\}^{p_{i j}}\right]+\sum_{(i, j) \notin I_{1}}\left[(i j)^{-s}\left\{f\left(q_{E}\left(x_{i j}-\ell\right)\right)\right\}^{p_{i j}}\right]\right. \\
& \quad \geq \frac{1}{n k} \sum_{(i, j) \in I_{1}}\left[(i j)^{-s}\left\{f\left(q_{E}\left(x_{i j}-\ell\right)\right)\right\}^{p_{i j}}\right] \\
& \quad \geq \epsilon \frac{1}{n k} C\left(\left\{(i, j) \in \mathbb{N} \times \mathbb{N}, i \leq n, j \leq k:\left[(n k)^{-s}\left\{f\left(q_{E}\left(x_{i j}-\ell\right)\right)\right\}^{p_{i j}}\right] \geq \epsilon\right\}\right)
\end{aligned}
$$

implies that $x=\left(x_{i j}\right)$ is statistically convergent to $\ell$.

Theorem 6.2. $w_{E, p, f, s}^{2} \cap \ell_{\infty}^{2}(E, p, f, s)=s_{E, p, f, s}^{2} \cap \ell_{\infty}^{2}(E, p, f, s)$, where $\ell_{\infty}^{2}(E, p, f, s)=\left\{x=\left(x_{n k}\right) \in S_{2}(E): x_{n k} \in E\right.$ and $\left.\left((n k)^{-s}\left\{f\left(q_{E}\left(x_{n k}\right)\right)\right\}^{p_{n k}}\right) \in \ell_{\infty}^{2}\right\}$.

Proof. Let $x=\left(x_{i j}\right) \in s_{E, p, f, s}^{2} \cap \ell_{\infty}^{2}(E, p, f, s)$ and let

$$
I_{2}(\epsilon)=\left\{(i, j), i \leq n, j \leq k:\left[(i j)^{-s}\left\{f\left(q_{E}\left(x_{i j}-\ell\right)\right\}^{p_{i j}}\right)\right] \geq \frac{\epsilon}{2}\right\}
$$

Let

$$
T=\sup _{i, j}\left[(i j)^{-s}\left\{f\left(q_{E}\left(x_{i j}-\ell\right)\right)\right\}^{p_{i j}}\right] .
$$

Since $x=\left(x_{i j}\right)$ is bounded statistically convergent, we can choose $\mathrm{N}$ such that for all $n, k \geq N$,

$$
\frac{1}{n k} C\left(\left\{(i, j) i \leq n, j \leq k:\left[(i j)^{-s}\left\{f\left(q_{E}\left(x_{i j}-\ell\right)\right)\right\}^{p_{i j}}\right] \geq \frac{\epsilon}{2}\right\}\right)<\frac{\epsilon}{2 T} .
$$


Thus

$$
\begin{aligned}
& \frac{1}{n k} \sum_{i=1}^{n} \sum_{j=1}^{k}\left[(I j)^{-s}\left\{f\left(q_{E}\left(x_{i j}-\ell\right)\right)\right\}^{p_{i j}}\right] \\
& =\frac{1}{n k} \sum_{(i, j) \in I_{2}(\epsilon)}\left[(I j)^{-s}\left\{f\left(q_{E}\left(x_{i j}-\ell\right)\right)\right\}^{p_{i j}}\right]+\frac{1}{n k} \sum_{(i, j) \notin I_{2}(\epsilon)}\left[(I j)^{-s}\left\{f\left(q_{E}\left(x_{i j}-\ell\right)\right)\right\}^{p_{i j}}\right] \\
& <\frac{1}{n k} n k \frac{\epsilon}{2 T} T+\frac{1}{n k} n k \frac{\epsilon}{2}=\epsilon
\end{aligned}
$$

Hence $x=\left(x_{i j}\right)$ is strongly $\left(p_{i j}\right)$-Cesáro-type summable to $\ell$.

We have proved more generalized form of some well known results of Mursaleen et al. [2003] regarding statistical convergence as follows:

Theorem 6.3. A vector valued double sequence $x=\left(x_{i j}\right)$ in $F^{2}(E, p, f, s)$ is statistically convergent to a number $\ell$ if and only if there exists a subset $R=\{(i, j)\} \subseteq N \times N$, $i, j=1,2, \ldots$ such that $\delta_{2}(R)=1$ and

$$
\lim _{\substack{i, j \rightarrow \infty \\(i, j) \in R}} q_{E}\left(x_{i j}-\ell\right)=0 .
$$

Proof. Let $x=\left(x_{i j}\right)$ be statistically convergent to $\ell$.

Let

$$
R_{\eta}=\left\{(i, j) \in \mathbb{N} \times \mathbb{N}:\left[(i j)^{-s}\left\{f\left(q_{E}\left(x_{i j}-\ell\right)\right)\right\}^{p_{i j}}\right] \geq 1 / \eta\right\}
$$

and

$$
T_{\eta}=\left\{(i, j) \in \mathbb{N} \times \mathbb{N}:\left[(i j)^{-s}\left\{f\left(q_{E}\left(x_{i j}-\ell\right)\right)\right\}^{p_{i j}}\right]<1 / \eta\right\}
$$

$(\eta=1,2, \ldots)$

Then $\delta_{2}\left(R_{\eta}\right)=0$. Again, $\left(T_{i}\right)$ is a sequence of sets such that $T_{i} \supseteq T_{i+1}$ and $\delta_{2}\left(T_{\eta}\right)=$ $1, \eta=1,2, \ldots$. Now, we have to show that for $(i, j) \in T_{\eta},\left(x_{i j}\right)$ is convergent to $\ell$.

Now, if possible, let $x=\left(x_{i j}\right)$ be not convergent to $\ell$, for all $(i, j) \in T_{\eta}$.

Then there is a $\epsilon>0$ such that, for infinitely many $i, j$,

$$
\left[(i j)^{-s}\left\{f\left(q_{E}\left(x_{i j}-\ell\right)\right)\right\}^{p_{i j}}\right] \geq \epsilon
$$

Let

$$
T_{\epsilon}=\left\{(i, j):\left[(i j)^{-s}\left\{f\left(q_{E}\left(x_{i j}-\ell\right)\right)\right\}^{p_{i j}}\right]<\epsilon\right\}
$$

where $\epsilon>1 / \eta$. Then $\delta_{2}\left(T_{\epsilon}\right)=0$.

Since $T_{\eta} \subseteq T_{\epsilon}$, it follows that $\delta_{2}\left(T_{\eta}\right)=0$, a contradiction. Thus $x=\left(x_{i j}\right)$ is convergent to $\ell$.

Conversely, suppose that there exists a subset $\mathrm{R}=\{(\mathrm{i}, \mathrm{j})\} \subseteq N \times \mathrm{N}$ such that $\delta_{2}(\mathrm{R})=1$ and

$$
\lim _{i, j \rightarrow \infty} q_{E}\left(x_{i j}-\ell\right)=0 .
$$


So there exists a +ve integer $N_{0}$ such that for every $\epsilon>0$,

$$
\left[(i j)^{-s}\left\{f\left(q_{E}\left(x_{i j}-\ell\right)\right)\right\}^{p_{i j}}\right]<\epsilon
$$

for all $i, j \geq N_{0}$.

Now,

$$
\begin{aligned}
R_{\epsilon} & =\left\{(i, j) \in \mathbb{N} \times \mathbb{N}:\left[(i j)^{-s}\left\{f\left(q_{E}\left(x_{i j}-\ell\right)\right)\right\}^{p_{i j}}\right] \geq \epsilon\right\} \\
& \subseteq \mathbb{N} \times \mathbb{N}-\left\{\left(n_{N_{0}+1}, k_{N_{0}+1}\right),\left(n_{N_{0}+2}, k_{N_{0}+2}\right), \ldots,\right\}
\end{aligned}
$$

Then

$$
\delta_{2}\left(R_{\epsilon}\right) \leq 1-\delta_{2}\left(\left\{\left(n_{N_{0}+1}, k_{N_{0}+1}\right),\left(n_{N_{0}+2}, k_{N_{0}+2}\right), \ldots,\right\}\right)=1-1=0
$$

Consequently $\mathrm{x}$ is statistically convergent to $\ell$.

Coroloary 6.1. If $s_{E, f, p, s}^{2}-\lim x_{i j}=\ell$, then there exists a sequence $y=\left(y_{i j}\right)$ such that $\lim _{i, j} y_{i j} \stackrel{q_{E}}{=} \ell$ and $\delta_{2}\left(\left\{(i, j): x_{i j} \neq y_{i j}\right\}\right)=1$ i.e., $x_{i j}=y_{i j}$ for all most all $i, j$.

Theorem 6.4. The set $s_{E, f, p, s}^{2} \cap \ell_{\infty}^{2}(E, p, f, s)$ is a closed linear subspace of the normed linear space $\ell_{\infty}^{2}(E, p, f, s)$.

Proof. Let $x^{(m n)}=\left(x_{i j}^{(m n)}\right)$ be any Cauchy sequence in the space $s_{E, f, p, s}^{2} \cap \ell_{\infty}^{2}(E, p$, $f, s)$. Let $x^{(m n)} \rightarrow x \in \ell_{\infty}^{2}(E, p, f, s)$. Since $x^{(m n)} \in s_{E, f, p, s}^{2}$, there exist $a_{m n} \in E$ such that $s_{E, f, p, s}^{2}-\lim _{i, j} x_{i j}^{(m n)}=a_{m n}$ for $m, n=1,2, \ldots$.

Since $x^{(m n)} \rightarrow x$, for every $\epsilon>0$, there exist a positive integer $n_{0} \in \mathbb{N}$ such that

$$
g\left(x^{(m n)}-x^{(p q)}\right)<\frac{\epsilon}{3}
$$

for every $m, p \geq n_{0}, n, q \geq n_{0}$, where $g$ denotes the norm. and

By Theorem 6.3. there exist subsets $K_{1}$ and $K_{2}$ of $\mathbb{N} \times \mathbb{N}$ with $\delta_{2}\left(K_{1}\right)=1=\delta_{2}\left(K_{2}\right)$

$$
\lim _{\substack{i, j \rightarrow \infty \\(i, j) \in K_{1}}} x_{i j}^{(m n)} \underline{\underline{g}} a_{m n} \text { and } \lim _{\substack{i, j \rightarrow \infty \\(i, j) \in K_{2}}} x_{i j}^{(p q)} \underline{\underline{g}} a_{p q}
$$

We choose $\left(k_{1}, k_{2}\right) \in K_{1} \cap K_{2}\left(\right.$ where $\left.\delta_{2}\left(K_{1} \cap K_{2}\right)=1\right)$.

Then by (6.2) we have

$$
g\left(x_{k_{1} k_{2}}^{(m n)}-a_{m n}\right)<\frac{\epsilon}{3}
$$

and

$$
g\left(x_{k_{1} k_{2}}^{(p q)}-a_{p q}\right)<\frac{\epsilon}{3}
$$


Therefore for each $m, p \geq n_{0}$ and $n, q \geq n_{0}$, using (6.1), (6.3) and (6.4) we have

$$
\begin{aligned}
g\left(a_{p q}-a_{m n}\right) & \leq g\left(x_{k_{1} k_{2}}^{(m n)}-a_{m n}\right)+g\left(x_{k_{1} k_{2}}^{(p q)}-a_{p q}\right)+g\left(x_{k_{1} k_{2}}^{(m n)}-x_{k_{1} k_{2}}^{(p q)}\right) \\
& <\frac{\epsilon}{3}+\frac{\epsilon}{3}+\frac{\epsilon}{3}=\epsilon
\end{aligned}
$$

Hence the sequence $\left(a_{m n}\right)$ is a Cauchy sequence in $E$. Since $E$ is a Banach space, it is complete. Let

$$
\lim _{m, n} q_{E}\left(a_{m n}\right)=a
$$

We will show that $x$ is statistically convergent to $a$.

Since $x^{(m n)}$ is convergent to $x$, for every $\epsilon>0$, there exist $N_{1}(\epsilon)$ such that for $i, j \geq N_{1}(\epsilon)$,

$$
g\left(x_{i j}^{(m n)}-x_{i j}\right)<\frac{\epsilon}{3}
$$

Also since (6.5) holds, we have for every $\epsilon>0$, there exist $N_{2}(\epsilon)$ such that for $i, j \geq N_{2}(\epsilon)$,

$$
g\left(a_{m n}-a\right)<\frac{\epsilon}{3}
$$

Again since $s_{E, f, p, s}^{2}-\lim _{i, j} x_{i j}^{(m n)}=a_{m n}$, there exists a set $R=\{(i, j)\} \subseteq \mathbb{N} \times \mathbb{N}$, $i, j=1,2, \ldots$ such that $\delta_{2}(R)=1$ and for every $\epsilon>0$, there exist $N_{3}(\epsilon)$ such that for $i, j \geq N_{3}(\epsilon),(i, j) \in R$,

$$
g\left(x_{i j}^{(m n)}-a_{m n}\right)<\frac{\epsilon}{3}
$$

Let

$$
N(\epsilon)=\max \left(N_{1}(\epsilon), N_{2}(\epsilon), N_{3}(\epsilon)\right)
$$

Then for every $\epsilon>0$, there exist $N(\epsilon)$ such that for $i, j \geq N(\epsilon),(i, j) \in R$,

$$
g\left(x_{i j}-a\right) \leq g\left(x_{i j}-x_{i j}^{(m n)}\right)+g\left(x_{i j}^{(m n)}-a_{m n}\right)+g\left(a_{m n}-a\right)<\frac{\epsilon}{3}+\frac{\epsilon}{3}+\frac{\epsilon}{3}=\epsilon
$$

Therefore $x$ is statistically convergent to $a$, i.e., $x \in s_{E, f, p, s}^{2} \cap \ell_{\infty}^{2}(E, p, f, s)$. Hence the proof.

Theorem 6.5. The set $s_{E, p, f, s}^{2} \cap \ell_{\infty}^{2}(E, p, f, s)$ is a nowhere dense in $\ell_{\infty}^{2}(E, p, f, s)$.

Proof. It is shown by T. Neubrum et al. [10] that every closed subspace of an arbitrary linear normed space $S$ different from $S$ is a nowhere dense set in $S$ and using Theorem 5.5.4, it is enough to show that $s_{E, p, f, s}^{2} \cap \ell_{\infty}^{2}(E, p, f, s) \neq \ell_{\infty}^{2}(E, p, f, s)$ in order to establish our claim.

Let us take $F^{2}=\mathbb{R} \times \mathbb{R}, E=\mathbb{R}, p_{i j}=1$. Let $x=\left(x_{i j}\right)$ be such that

$$
x_{i j}= \begin{cases}1 & \text { if } i \text { and } j \text { are even } \\ 0 & \text { otherwise }\end{cases}
$$


Let $f(x)=x$ and $s=0$. Then $x=\left(x_{i j}\right)$ is not statistically convergent, but it is bounded. Hence the result.

Definition 6.5. A sequence $x=\left(x_{i j}\right)$ is said to be statistically Cauchy if for any given $\epsilon>0$, there exist $N_{1}(\epsilon)$ and $N_{2}(\epsilon)$ such that for all $i, r \geq N_{1}$ and $j, t \geq N_{2}$,

$$
\left\{(i, j), i \leq n, j \leq k:\left[(i j)^{-s}\left\{f\left(q_{E}\left(x_{i j}-x_{r t}\right)\right)\right\}^{p_{i j}}\right] \geq \epsilon\right\}
$$

has double natural density zero.

Theorem 6.6. A sequence $x=\left(x_{i j}\right)$ in $F^{2}(E, p, f, s)$ is statistically convergent if and only if it is statistically Cauchy.

Proof. Let us assume that $x=\left(x_{i j}\right)$ in $F^{2}(E, p, f, s)$ is statistically convergent to $\ell$. Then for any given $\epsilon>0$, the set

$$
\left.\left\{(i, j), n \leq u, k \leq v:\left[\left((i j)^{-s}\left\{f\left(q_{E}\left(x_{i j}-\ell\right)\right)\right\}^{p_{i j}}\right)\right]\right) \geq \epsilon\right\}
$$

has double natural density zero.

Let $N_{1}$ and $N_{2}$ be so chosen that

$$
\left[\left(N_{1} N_{2}\right)^{-s}\left\{f\left(q_{E}\left(x_{N_{1} N_{2}}-\ell\right)\right)\right\}^{p_{i j}}\right] \geq \epsilon .
$$

Now,

$$
\begin{gathered}
\left\{(i, j), n \leq u, k \leq v:\left[(i j)^{-s}\left\{f\left(q_{E}\left(x_{i j}-x_{N_{1} N_{2}}\right)\right)\right\}^{p_{i j}}\right] \geq \epsilon\right\} \\
\subseteq\left\{(i, j), n \leq u, k \leq v:\left[(i j)^{-s}\left\{f\left(q_{E}\left(x_{i j}-\ell\right)\right)\right\}^{p_{i j}}\right] \geq \epsilon\right\} \\
\cup\left\{(i, j), n \leq u, k \leq v:\left[(i j)^{-s}\left\{f\left(q_{E}\left(x_{N_{1} N_{2}}-\ell\right)\right)\right\}^{p_{i j}}\right] \geq \epsilon\right\}
\end{gathered}
$$

Hence

$$
\begin{aligned}
& \delta_{2}\left(\left\{(i, j), n \leq u, k \leq v:\left[(i j)^{-s}\left\{f\left(q_{E}\left(x_{i j}-x_{N_{1} N_{2}}\right)\right)\right\}^{p_{i j}}\right] \geq \epsilon\right\}\right) \\
& \leq \delta_{2}\left(\left\{(i, j), n \leq u, k \leq v:\left[(i j)^{-s}\left\{f\left(q_{E}\left(x_{i j}-\ell\right)\right)\right\}^{p_{i j}}\right] \geq \epsilon\right\}\right) \\
& \quad+\delta_{2}\left(\left\{(i, j), n \leq u, k \leq v:\left[(i j)^{-s}\left\{f\left(q_{E}\left(x_{N_{1} N_{2}}-\ell\right)\right)\right\}^{p_{i j}}\right] \geq \epsilon\right\}\right) \\
& \quad=0
\end{aligned}
$$

where $\delta_{2}(A)$ denotes the double natural density of the set $A$. Thus $x=\left(x_{i j}\right)$ is statistically Cauchy sequence.

Conversely, let $x=\left(x_{i j}\right)$ be statistically Cauchy sequence, but not statistically convergent. Then

$$
\left.\delta_{2}\left(\left\{(i, j), i \leq n, j \leq k:\left[(i j)^{-s}\left\{f\left(q_{E}\left(x_{i j}-x_{N_{1} N_{2}}\right)\right)\right\}^{p_{i j}}\right]\right) \geq \epsilon\right\}\right)=0
$$


i.e.,

$$
\left.\delta_{2}\left(\left\{(i, j), i \leq n, j \leq k:\left[(i j)^{-s}\left\{f\left(q_{E}\left(x_{i j}-x_{N_{1} N_{2}}\right)\right)\right\}^{p_{i j}}\right]\right)<\epsilon\right\}\right)=1 .
$$

So, in particular,

$$
\left[(i j)^{-s}\left\{f\left(q_{E}\left(x_{i j}-x_{N_{1} N_{2}}\right)\right)\right\}^{p_{i j}}\right] \leq 2\left[(i j)^{-s}\left\{f\left(q_{E}\left(x_{i j}-\ell\right)\right)\right\}^{p_{i j}}\right]<\epsilon
$$

holds if

$$
\left[(i j)^{-s}\left\{f\left(q_{E}\left(x_{i j}-\ell\right)\right)\right\}^{p_{i j}}\right]<\epsilon / 2
$$

If possible, let $x=\left(x_{i j}\right)$ be not statistically convergent. Then

$$
\left.\delta_{2}\left\{(i, j), i \leq n, j \leq k:\left[\left((i j)^{-s}\left\{f\left(q_{E}\left(x_{i j}-\ell\right)\right)\right\}^{p_{i j}}\right]\right)<\epsilon\right\}\right)=0 .
$$

Therefore the set

$$
\left.\delta_{2}\left(\left\{(i, j), i \leq n, j \leq k:\left[(i j)^{-s}\left\{f\left(q_{E}\left(x_{i j}-x_{N_{1} N_{2}}\right)\right)\right\}^{p_{i j}}\right]\right)<\epsilon\right\}\right)=0
$$

which contradicts (6.6). Hence $x=\left(x_{i j}\right)$ is statistically convergent to $\ell$.

\section{Summary and Conclusion}

Considering $F^{2}=\mathbb{R}^{2}, E=\mathbb{R}, p_{i j}=1, f=I, s=0$, all the results of Mursaleen et al. 9] regarding statistical convergence can be obtained from these results. Moreover, if we take $p=\left(p_{i j}\right)$ to be a sequence of constant terms, say, $p_{i j}=p$, where $0<p<1$, then $M=1$ and restricting $F^{2}=E=\mathbb{R}, f=I, s=0$, our $\left(p_{i j}\right)$-Cesáro-type summability reduces to p-Cesáro summability defined by Mursaleen et al. [9] .

\section{References}

[1] A. Pringsheim, Zur Theorie der zweifach unendlichen Zahlenfolgen, Math. Ann. 53 (1900), 289-321.

[2] B. C. Tripathy, Statistically convergent double sequences, Tamkang J. Math. 34(2003), 231237.

[3] T. Bilgin, The sequence space $\ell\left(p, f^{v}, q, s\right)$, Journal of Faculty of Education, 1(1), (1994), 73-82.

[4] A. Gökhan, R. Çolak, The double sequence spaces $c_{2}^{P}(p)$ and $c_{2}^{P B}(p)$, Appl. Math. Comp. 157 (2004), 491-501.

[5] A. Gökhan and R. Çolak, Double sequence space $\ell_{2}^{\infty}(p)$, Appl. Math. Comp., 160(2005), 147-153.

[6] D. Ghosh and P. D. Srivastava, On some vector valued sequence spaces defined using a modulus function, Indian J. Pure Appl. Math, 30(1999), 819-826. 
[7] I. J. Maddox, Sequence spaces defined by a modulus, Proc. Camb. Phil. Soc. 100(1986), 161-166.

[8] F. Moricz, Tauberian theorems for Cesaro summable double sequences, Studia Math. 110(1994), 83-96.

[9] E. Mursaleen and O. H. H. Edely, Statistical convergence of double sequences, J. Math. Anal. Appl., 288(2003), 223-231.

[10] T. Neubrum, J. Smital and T. Sàlat, On the structure of the space $M(0,1)$, Rev. Roumaine Math. Pures Appl., 13(1968), 337-386.

[11] E. Özturk and T. Bilgin, Strongly summable sequence spaces defined By a modulus, Indian J. Pure Appl. Math., 25 (2004), 621-625.

[12] A. Ratha (P. D. Srivastava), I. I. T, Kharagpur, 1993, Ph.D Thesis.

[13] Tripathy, B. C., Generalized Köthe-Toeplitz dual of some double sequence spaces, communicated, (2004).

Department of Mathematics, Indian Institute of Technology, Kharagpur-721302, India.

E-mail: abasu@maths.iitkgp.ernet.in

E-mail: pds@maths.iitkgp.ernet.in 